\title{
S-G-5. The Resuscitation of the Central Nervous System
}

\author{
Tatsuyuki Kudo, Shoji Ishimori, Mitsuo Hattori, \\ and Haruo Suzuk I \\ Div. of Neurological Surgery, Dept. of Surgery, School of Medicine, Keio Univ. \\ Haruo Matsuyama \\ Dept. of Pathology, School of Medicine, Keio Univ.
}

The canine brain function was disappeared under the extremely profound hypothermia, and was preserved for arbitrary period and was resuscitated by the rewarming. The recovery of the nerve cell was evaluated by the reappearance of the brain function as the indication of the recovery of the various vital signs, such as corneal reflex, cilliary reflex, respiratory movements, responce to painful stimuli and EEG activities. The histopathological changes in the brain which were occurred throughout these procedure were observed on the isolated brain cooling and on the decorporated head cooling indepandently.

There was no noticeable neurological deficit in the group of the recovered dogs with the complete circulatory arrest of $1 \mathrm{hr}$. under the isolated brain cooling. As for the histo-pathological changes of the survived dogs, the scattered minimal localized changes of the cerebral cortex and the decreased numbers of the Purkinje cells were observed. The authors concluded that the brain regained normal neurological function inspite of those pathological changes.

The decorporated head cooling was performed to determine the limitation of the safe period of the circulatory arrest. And the resuscitation was achieved after $6 \mathrm{hrs}$. of the circulatory arrest. The similar histological changes were observed in the resuscitated brain as were seen in the $1 \mathrm{hr}$. of the circulatory arrest of the isolated brain cooling.

The specimens were taken from the brain of the each stage of the cooling, the preservation and the rewarming. The above described pathological changes of the brain have mainly occurred in the rewarming stage. The cause of these changes was discussed.

It would be strongly possible to resuscitate the cooled brain after the longer period of the circulatory arrest if this method could be further developed. 